\title{
Réabsorption du sel et sécrétion du potassium par le néphron distal
}

> L'étude d'une forme mendélienne rare d'hypertension artérielle, l'hypertension hyperkaliémique familiale (FHHt), a permis des avancées remarquables dans la compréhension des mécanismes de régulation du transport rénal du chlorure de sodium. Chez quelques patients, cette pathologie est due à des mutations touchant les gènes codant WNKl et WNK4, deux sérine-thréonine kinases de la famille WNK (with no lysine $[K])$. Les signes cliniques associés à la FHHt résultent, entre autres, de l'hyperactivité du co-transporteur $\mathrm{Na}^{+}-\mathrm{Cl}^{-}$, NCC. De nombreuses équipes se sont intéressées à la régulation de NCC par WNKl et WNK4. Cependant, les données obtenues étaient très souvent contradictoires. Récemment, deux de nos études ont permis d'expliquer en partie ces controverses et d'établir un nouveau modèle de régulation de NCC par les kinases de la famille WNK. <

\section{WNK1-WNK4 et hypertension hyperkaliémique familiale}

L'hypertension artérielle essentielle (HTA) est une maladie complexe, causée par une combinaison de facteurs génétiques et environnementaux. L'un des facteurs de risque les plus connus est l'exposition chronique à un régime trop riche en chlorure de sodium [1,2] mais les mécanismes responsables de cette sensibilité de la pression artérielle au sel n'ont pas encore été complétement élucidés. Cependant, grâce à une modélisation mathématique complexe, A.C. Guyton proposait en 1972 qu'un défaut de transport ionique dans le néphron était impliqué dans toutes les formes d'HTA [3].

Une des stratégies d'étude utilisées pour identifier de nouveaux facteurs mis en jeu dans la régulation de la pression artérielle est l'analyse génétique de formes caricaturales, rares et mendéliennes d'hypertension artérielle. L'hypertension hyperkaliémique familiale ( FHHt), également connue sous le nom de syndrome de Gordon ou pseudohypoaldostéronisme de type 2, est

\section{Vision nouvelle du rôle régulateur des kinases de la famille WNK}

Chloé Rafael ${ }^{1-3}$, Maria Chavez-Canales ${ }^{1,2}$, Juliette Hadchouel ${ }^{1,2}$

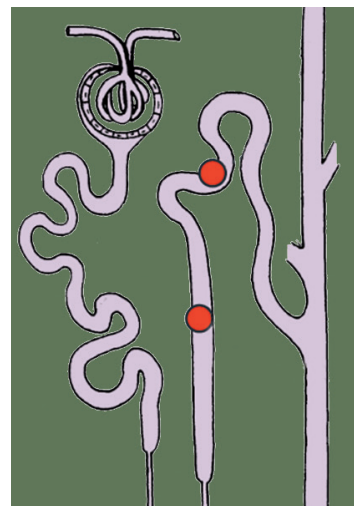

${ }^{1}$ Inserm UMR970, Paris Centre de Recherche cardiovasculaire, 56, rue Leblanc, 75015 Paris, France :

${ }^{2}$ Université Paris-Descartes, Sorbonne Paris Cité, Paris, France ;

${ }^{3}$ Université Paris-Diderot, Sorbonne Paris Cité, Paris, France.

juliette.hadchouel@inserm.fr

I'un de ces syndromes [4]. Les patients atteints de FHHt présentent une hypertension modérée, une hyperkaliémie ${ }^{1}$ et une acidose métabolique hyperchlorémique ${ }^{2}$. L'ensemble de ces troubles est corrigé par une faible dose de diurétiques thiazidiques ${ }^{3}[33](\rightarrow)$, qui inhibent le co-transporteur $\mathrm{Na}^{+}-\mathrm{Cl}^{-} \mathrm{NCC}\left(\mathrm{Na}^{+}-\mathrm{Cl}^{-}\right.$cotransporter) exprimé dans le segment du néphron appelé tubule contourné distal (DCT) (Figure 1).

$\rightarrow$ Voir la Nouvelle de D. Eladari et al., $\mathrm{m} / \mathrm{s} \mathrm{n} \mathrm{n}^{\circ}$ 5, mai 2010, Cette sensibilité aux diurétiques thiazidiques a page 549

laissé supposer que la $\mathrm{FHHt}$ avait pour cause une activation du cotransporteur NCC. Des mutations inactivatrices de NCC sont responsables du syndrome de Gitelman, un syndrome miroir de la FHHt (qui se caractérise entre autres par une alcalose hypokaliémique). Cependant, aucune mutation du gène codant NCC (SLC12A3 [solute carrier family 12, member 3]) n'a été identifiée chez les patients atteints de $\mathrm{FHHt}$. De plus, les analyses de liaisons génétiques n'ont pas permis de mettre en évidence une association significative entre la $\mathrm{FHHt}$ et le bras court du chromosome 16, où est localisé le gène SLC12A3.

\footnotetext{
${ }^{1}$ Excès de potassium dans le sang.

${ }^{2}$ Baisse du pH sanguin associée à un excès de chlorure.

${ }^{3}$ Les thiazidiques ont été développés initialement pour leur propriété d'inhibition de l'anhydrase carbonique mais leur effet diurétique s'est révélé supérieur. Ils sont constitués par la fusion entre un noyau de
} benzène et un noyau de thiadiazine (comportant 2 atomes d'azote et $l$ atome de soufre). 


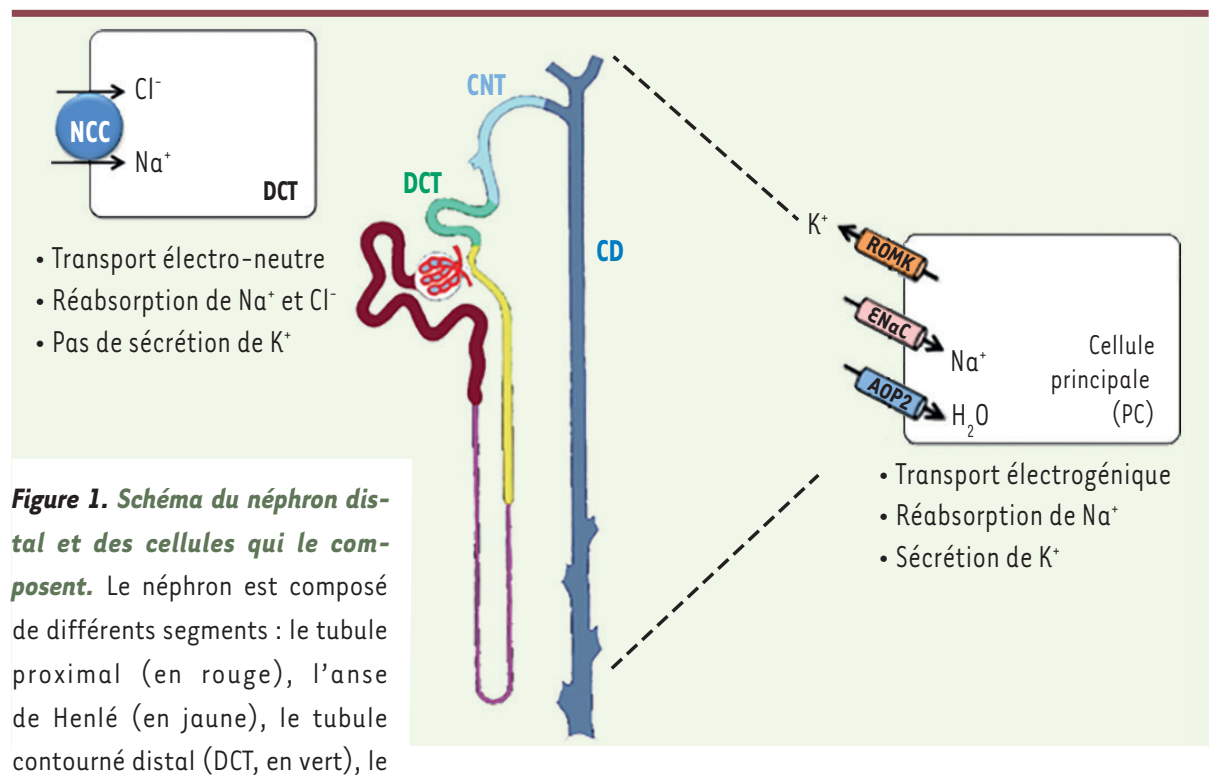
contourné distal (DCT, en vert), le tubule connecteur (CNT, en bleu clair) et le canal collecteur (CD, en bleu foncé). Ces trois derniers segments forment le néphron distal sensible à l'aldostérone. Le DCT est composé d'un seul type cellulaire qui exprime à sa membrane apicale le transporteur $\mathrm{Na}^{+}-\mathrm{Cl}^{-}, \mathrm{NCC}$. Ce transporteur permet la réabsorption électroneutre de $\mathrm{Na}^{+}$et $\mathrm{Cl}^{-}$, sans sécrétion de $\mathrm{K}^{+}$. À l'inverse, les cellules principales du CD réabsorbent le $\mathrm{Na}^{+}$grâce au $\varepsilon \mathrm{NaC}$ (epithelial sodium channel). Cette réabsorption génère un gradient électrochimique favorisant la sécrétion de $\mathrm{K}^{+}$par le canal ROMK (renal outer medullary potassium channel). AQP2 : aquaporine 2.

\section{La controverse WNK1}

WNK1, activateur de SPAK ou inhibiteur de WNK4?

Les premières études portant sur la régulation de NCC par les WNK ont été menées in vitro en utilisant des œufs de xénope ${ }^{4}$ ou des cultures de lignées cellulaires. Dans ces modèles, la surexpression de WNK4 inhibe l'expression membranaire de NCC et donc son activité $[9,10]$. Ce mode de régulation dépend de l'activité kinase de WNK4 puisqu'un mutant WNK4-kinase dead, qui ne présente plus d'activité kinase, n'est plus capable d'inhiber NCC.

Les mécanismes par lesquels WNKl régule NCC sont longtemps restés controversés. Deux isoformes de WNKl ont été décrites [11]: L-WNKl (long-WNK1), qui est ubiquitaire et possède une activité catalytique, et KS-WNKI
En 2001, les premières mutations responsables de la FHHt ont été identifiées [5]. Elles sont localisées dans les gènes qui codent deux membres de la famille des sérine thréonine kinases WNK (with no lysine [K]), WNKl et WNK4, qui sont exprimées dans le rein. Le nom étrange de ces kinases est dû au remplacement de la lysine catalytique, présente dans le sous-domaine II de la majorité des protéine kinases connues, par une cystéine [6]. De nombreuses équipes ont étudié la régulation de NCC par les kinases WNKl et WNK4 [34] $(\rightarrow)$.

Cependant, les résultats obtenus étaient souvent contradictoires et un modèle clair et unique expliquant le mécanisme de régulation de l'expression et de l'activité du cotranspor- $\rightarrow$ Voir la Synthèse de J. Hadchouel et al., $m / s n^{\circ} 1$, janvier 2005 , page 55 teur NCC par les WNK n'a jamais pu être établi. En effet, en fonction des modèles utilisés, WNK4 et WNKI présentaient, dans certaines études, un rôle activateur de NCC et, dans d'autres, un rôle inhibiteur (voir plus loin). Deux publications récentes de notre équipe ont permis de commencer à expliquer ces contradictions [7, 8]. La première démontre que les résultats contradictoires des études portant sur la régulation de NCC par WNKl provenaient de l'utilisation d'un ADN complémentaire (ADNc) de WNKl présentant une mutation inactivatrice [8]. La deuxième montre que les effets activateurs et inhibiteurs de WNK4 sur la régulation de NCC sont en fait présents au sein d'une même cellule et qu'ils sont modulés par la concentration intracellulaire de chlorure [7]. Dans cette revue, nous reviendrons sur ces récentes découvertes et nous proposerons un nouveau modèle pour expliquer le rôle que jouent WNKl et WNK4 dans la régulation du transport de $\mathrm{NaCl}$ et de potassium dans le rein.
(kidney-specific-WNKI), qui est une forme tronquée de la protéine exprimée spécifiquement dans la partie distale du néphron et qui est dépourvue d'activité kinase. Dans la suite, nous nous intéresserons essentiellement à L-WNKl qui est l'isoforme impliquée dans la FHHt [12].

Plusieurs études avaient montré que la surexpression de L-WNKL, seul, dans l'œuf de xénope ou les cellules rénales MDCK (Madin-Darby canine kidney) ne modifiait pas l'activité de NCC $[10,13]$. Pourtant, L-WNKl peut activer NCC par inhibition de WNK4. Parallèlement, des études biochimiques avaient montré que L-WNKl pouvait activer NCC indépendamment de WNK4. En effet, L-WNKI phosphoryle la kinase SPAK (Ste20-related proline-alanine rich kinase) qui, elle-même, phosphoryle et active NCC [14]. Deux voies de régulation de NCC par L-WNKl étaient donc possibles.

Afin de déterminer la ou les voies impliquées in vivo, nous avons utilisé un modèle de souris génétiquement modifiées. L'inactivation du gène $L-W N K I$ provoque la mort des embryons avant le $13^{e}$ jour de développement [15]. Il est donc impossible d'étudier dans ce modèle la régulation de NCC par la kinase. Nous avons donc

\footnotetext{
${ }^{4}$ Le xénope est un amphibien, eucaryote. II présente l'avantage de pondre beaucoup d'œufs, autant d'embryons larges et facilement manipulables. Ses embryons représentent un bon modèle d'étude du transport ionique, après surexpression des
} protéines d'intérêt. 
généré un modèle de surexpression de la protéine L-WNKl. Les mutations de WNKI impliquées dans la FHHt sont de larges délétions de l'intron 1 du gène. Elles provoquent une surexpression de la protéine L-WNKl dans les leucocytes des patients [5]. Nous avons éliminé le premier intron de WNKI chez la souris (souris $W N K 1^{+/ F H H t}$ ) et avons ainsi obtenu un modèle présentant l'ensemble des phénotypes associés à la FHHt [12]. Cette délétion intronique entraîne une augmentation de l'expression de L-WNKl dans le DCT et le tubule connecteur sans que l'expression de KS-WNKl ne soit modifiée. Comme attendu, l'abondance et la phosphorylation de la protéine NCC sont augmentées. Nous avons également observé une augmentation de la forme phosphorylée de SPAK à la membrane apicale des cellules du DCT. Des travaux plus récents, utilisant le même modèle murin $W N K 1^{+/ F H H t}$ mais qui n'exprime pas WNK4, ont confirmé la capacité de L-WNKl à activer l'expression et la phosphorylation de NCC, même en absence de WNK4 [8]. Ces études in vivo sont donc en faveur d'une activation de NCC par L-WNKI qui dépend de SPAK mais qui est indépendante de WNK4.

\section{La controverse WNK1 : une simple erreur technique!}

Afin de comprendre les divergences observées entre les résultats obtenus in vitro et ceux observés in vivo, nous avons à nouveau examiné les effets de L-WNKI en utilisant différents systèmes d'étude in vitro. Huit exons de WNKI (exons 4a, 8b, HSN2, 9, 11, 12, 26a et 26b) sont soumis à un épissage alternatif [16]. Chez l'homme et la souris, le variant $L-W N K l-\Delta l 1$, qui ne contient pas l'exon 11 , est le variant majoritaire dans le néphron (il représente environ $70 \%$ de tous les variants de L-WNKl) [16]. La grande majorité des études concernant la régulation de NCC par L-WNKl in vitro a paradoxalement été réalisée en utilisant le variant $L-W N K l-\Delta 11-12$, qui ne représente en fait, dans le rein, que $20 \%$ des transcrits L-WNKl. Nous avons donc étudié la régulation de NCC par l'ensemble des variants humains, en utilisant les cellules HEK293 (human embryonic kidney) 5 et l'œuf de xénope [8]. De façon surprenante, tous les variants de L-WNKl sont susceptibles, après transfection dans ces cellules, d'activer le cotransporteur NCC. Le variant conférant la plus grande activation est cependant L-WNKl- $\Delta 1$ l. L'absence d'activation de NCC par L-WNKl observée dans les études précédentes n'est donc pas le résultat de l'utilisation d'un « mauvais variant». Il est important de noter que ces travaux ont été réalisés avec le même $A D N c$ de $L-W N K l-\Delta 11-12$ que celui qui a été sous-cloné lors de l'identification de WNK1, en 2000, à partir d'une banque d'ADNc de cerveau de rat [6]. Pour notre étude, nous avons sous-cloné les différents variants à partir de tissus humains. L'analyse par séquençage des ADNc d'homme et de rat correspondant au variant L-WNKl- $\Delta 1$ l-12 a mis en évidence une mutation dans la partie C-terminale de la protéine du rat (p.Gly210Ser) par rapport à l'homme. Cette mutation est le résultat d'une erreur de lecture par la Taq polymérase ${ }^{6}$ réalisée lors du sous-clonage de L-WNKl à partir de la banque d'ADNc. Elle ne correspond pas à une variation d'espèce puisque le séquençage

${ }^{5}$ La lignée de cellules rénales embryonnaires humaines HEK293 est très utilisée pour les expériences de transfection de gène afin d'étudier la fonctionnalité de la protéine correspondante.

${ }^{6}$ La Taq polymérase est une ADN polymérase utilisée pour la duplication de l'ADN au cours de la réaction de PCR (polymerase chain reaction). du génome du rat met en évidence une glycine à cette position, comme dans toutes les autres espèces, et non une sérine. La mutation de la glycine en sérine altère l'activité du variant et empêche l'activation de NCC. Le remplacement du résidu sérine par une glycine permet de restaurer l'activation de NCC par L-WNKl- $\Delta 11-12$ de rat et, à l'inverse, la mutation de I'ADNc du variant humain prévient l'activation de NCC [8].

Ces résultats confirment donc les résultats obtenus avec les modèles murins. Ils valident que L-WNKl est un activateur puissant de NCC in vitro et in vivo. Ils montrent également l'importance d'utiliser le variant approprié pour étudier la fonction d'un gène dont le messager peut subir plusieurs épissages alternatifs et donner ainsi naissance à plusieurs variants de la protéine. En effet, dans notre étude, le niveau d'activation de NCC dépend du variant utilisé, le niveau le plus élevé étant obtenu avec le variant le plus exprimé dans le rein.

\section{La controverse WNK4 est due à des différences de chlorure intracellulaire}

Les études menées dans l'œuf de xénope ou les cellules en culture ont montré que WNK4 est un inhibiteur de NCC et que cette inhibition dépend de son activité kinase $[9$, 10]. Pourtant, les souris porteuses d'une inactivation de WNK4 présentent une diminution de l'abondance et de la phosphorylation de NCC [17]. WNK4 serait donc un activateur de NCC in vivo.

Une deuxième série d'expériences a remis en question les résultats obtenus in vitro. Les mutations identifiées dans le locus WNK4 chez les patients FHHt sont des mutations ponctuelles faux-sens ${ }^{7}$ [5]. Elles sont pratiquement toutes localisées dans un petit motif de la protéine d'une dizaine d'acides aminés, présent dans toutes les kinases de la famille WNK. Les conséquences fonctionnelles de ces mutations sont longtemps restées indéterminées. Ce motif est situé en aval d'un domaine coiled-coil, généralement décrit comme un motif responsable de l'oligomérisation des protéines (c'est-à-dire leur appariement) [18]. Ces mutations pourraient donc modifier l'interaction de WNK4 avec ses partenaires. Cette hypothèse a été confirmée grâce à l'identification de deux nouveaux gènes dont des mutations sont responsables de la FHHt [19, 20], KLHL3 (Kelch-like 3) et Cullin3 [35] $(\rightarrow)$. $(\rightarrow)$ Voir la Nouvelle de H. Louis-Dit-Picard et al., $m / s n^{\circ} 8-9$, août-septembre 2012, page 703
${ }^{7}$ La mutation faux-sens occasionne un changement au niveau d'un nucléotide d'un codon. Elle provoque une modification de l'acide aminé qui lui est associé. L'activité de la protéine traduite peut en être modifiée. 


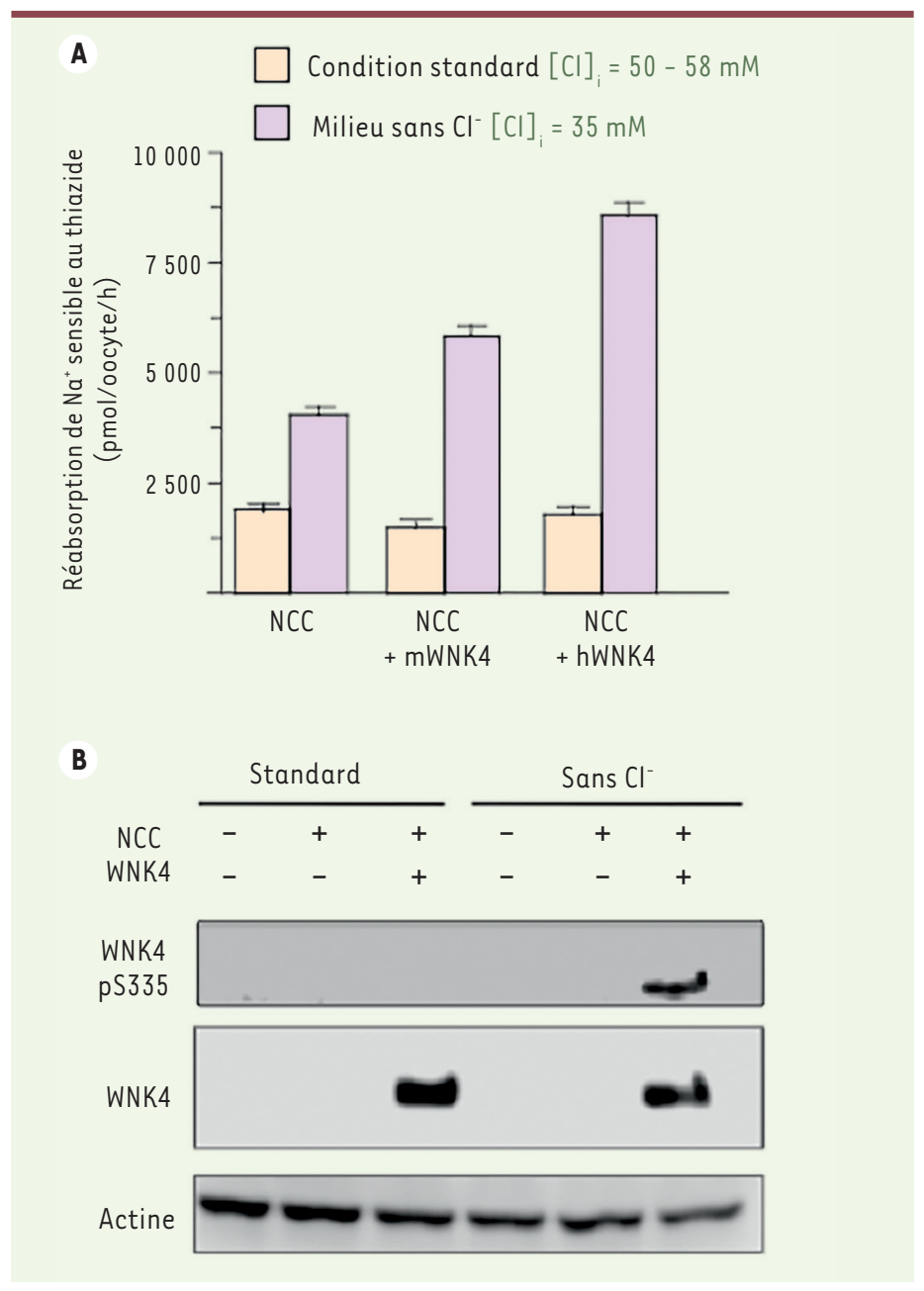

Figure 2. La régulation de NCC par WNK4 est modulée par la concentration intracellulaire en chlorure. A. Activité de $\mathrm{NCC}$ (transporteur $\mathrm{Na}^{+}-\mathrm{Cl}^{-}$) dans des oocytes de xénope surexprimant ou non WNK4. En conditions standard, la coexpression de NCC avec WNK4 de souris (mWNK4) ou d'homme (hWNK4) réduit légèrement ou ne modifie pas l'activité de NCC, respectivement (barres jaunes). Lorsque la concentration intracellulaire en chlorure $\left[\mathrm{Cl}^{-}\right]_{i}$ est abaissée par une incubation des oocytes dans un milieu hypotonique dépourvu de chlorure, l'activité de NCC est fortement stimulée par la surexpression de WNK4 (barres violettes). B. Phosphorylation de WNK4 en fonction de la concentration intracellulaire en chlorure $\left[\mathrm{Cl}^{-}\right]_{\mathrm{i}}$. En conditions standard, la phosphorylation de WNK4 sur la sérine en position 335 est indétectable par western blot. L'incubation des oocytes dans un milieu sans chlorure stimule fortement la phosphorylation de ce résidu (d'après Bazua-Valenti et al. [7]).

Les protéines produites par ces gènes forment un complexe ubiquitineligase dans lequel KLHL3 recrute les substrats pour qu'ils soient ubiquitinés, entraînant ainsi leur dégradation protéosomale ${ }^{8}$. WNK4 est l'un de ces substrats [21-23]. Les mutations touchant WNK4,

\footnotetext{
${ }^{8}$ Le système ubiquitine/protéasome joue un rôle majeur dans la protéolyse intracellulaire. De nombreux composants de ce système sont des complexes protéiques présentant dans une même structure, le protéasome, plusieurs activités enzymatiques, les enzymes d’ubiquitinylation.
}

responsables de la FHHt, empêchent son interaction avec $K L H L 3$, ce qui conduit à une augmentation de l'abondance de la protéine WNK4. Ces résultats ont été confirmés in vivo. L'expression de WNK4 est en effet augmentée dans le rein de souris porteuses d'une des mutations de WNK4 associées à la FHHt, et qui présentent toutes les signes de la FHHt [22]. La protéine WNK4 mutée aurait donc la même activité que la protéine sauvage. Cette hypothèse a été vérifiée in vivo. Des souris surexprimant la forme sauvage de WNK4 présentent en effet une augmentation de l'expression et de la phosphorylation de NCC ainsi qu'une FHHt [22]. L'ensemble de ces études permet donc de conclure que WNK4 est un activateur fort et essentiel de NCC in vivo. Comment expliquer alors que cette activation n'ait pas été observée in vitro ? L'explication la plus simple est celle d'une différence existant entre les systèmes utilisés pour les expériences in vitro (cellules HEK et œuf de xénope) et les cellules du DCT in situ, qui influencerait I'activité de WNK4. Plusieurs études avaient suggéré que l'activité des WNK était modulée par des changements de concentration ionique, notamment en chlorure et en potassium. Plus récemment, Piala et al. ont montré que L-WNKI est une kinase dont l'activité est sensible au chlorure [24]. Elle est en effet plus active lorsque la concentration en chlorure est faible. L'activation de L-WNK1 requiert la phosphorylation d'une sérine (S382) située dans la boucle d'activation du domaine kinase (activation loop) [25], qui est plus importante lorsque la concentration en chlorure est faible [24]. Des études de cristallographie ont mis en évidence que le chlorure se fixe au domaine kinase, maintenant la protéine dans une conformation inactive. Un résidu leucine (L379) joue un rôle crucial dans la formation du site de fixation pour le chlorure dans le domaine kinase. En effet, sa mutation en phénylalanine diminue la sensibilité de L-WNKl au chlorure [24].

La concentration en chlorure retrouvée dans les modèles cellulaires in vitro est plus élevée que celle observée dans les cellules du DCT (40-50 mM [7] versus 10-20 mM [26]). Les effets divergents de WNK4 sur NCC pourraient donc résulter de cette différence de concentration intracellulaire en chlorure $\left(\left[\mathrm{Cl}^{-}\right] \mathrm{i}\right)$ entre les deux systèmes cellulaires. Nous avons testé l'activité de NCC dans les ovocytes de xénope incubés dans un milieu standard $\left(\left[\mathrm{Cl}^{-}\right] \mathrm{i} \approx 50 \mathrm{mM}\right)$ ou dans un milieu hypotonique dépourvu de chlorure $\left(\left[\mathrm{Cl}^{-}\right] \mathrm{i} \approx 35 \mathrm{mM}\right)$. La co-injection de NCC et de WNK4 dans l'œuf de xénope en conditions standard résulte en une diminution de l'absorption de sodium par NCC. Cependant, lorsque la concentration intracellulaire de chlorure est abaissée expérimentalement, WNK4 active NCC (Figure 2). 


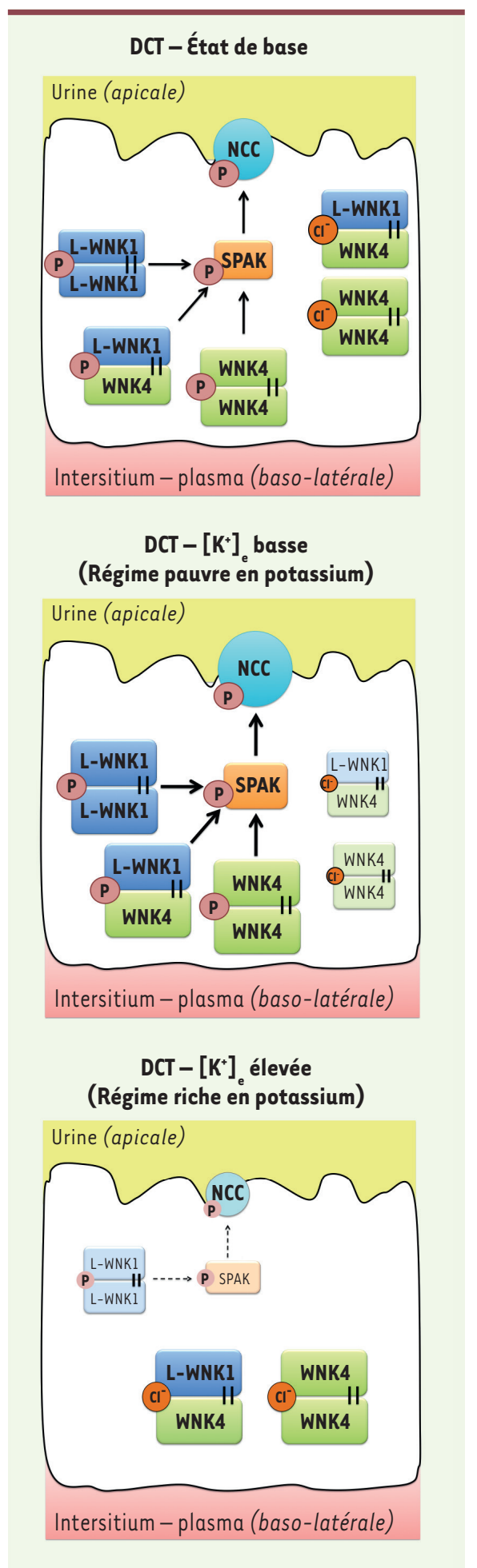

Figure 3. Modèle proposé pour la régulation de NCC par WNKI et WNK4. À l'état basal (panneau du haut), le niveau de phosphorylation de NCC (transporteur $\mathrm{Na}^{+}-\mathrm{Cl}^{-}$) est maintenu grâce à la phosphorylation de SPAK (Ste20-related proline-alanine rich kinase) par une partie des hétéromères L-WNK1/WNK4 et des homomères L-WNKL et WNK4, qui sont phosphorylés et actifs. Une autre partie des ces multimères est rendue inactive par la fixation des ions chlorure au niveau du domaine catalytique des kinases. Lors de l'ingestion d'un régime pauvre en potassium (panneau du milieu), la concentration intracellulaire de chlorure diminue, ce qui active un plus grand nombre de multimères L-WNK1/WNK4 et augmente la phosphorylation de SPAK puis de NCC et donc le nombre de cotransporteurs actifs. À l'inverse, lors de l'ingestion d'un régime riche en potassium (panneau du bas), l'augmentation de la concentration intracellulaire de chlorure inactive la plupart des multimères L-WNK1/WNK4, diminuant ainsi la phosphorylation de SPAK et de NCC, et donc le nombre de cotransporteurs à la membrane. Les barres noires entre les molécules de WNK symbolisent le domaine HQ (petit motif carboxy-terminal requis pour l'interaction des kinases WNK entre elles).

L'activation de WNK4, comme pour L-WNKl, dépend de la phosphorylation de sa boucle d'activation, qui augmente lorsque la concentration intracellulaire de chlorure diminue (Figure 2). La mutation de la leucine présente dans le site de fixation du chlorure permet à WNK4 d'activer NCC dans les conditions standard en absence de déplétion en chlorure. WNK4 est donc une kinase sensible au chlorure. Ces observations expliquent donc vraisemblablement la dualité des effets activateurs et inhibiteurs que WNK4 peut avoir sur NCC en fonction de la concentration intracellulaire de chlorure.

Le fait que L-WNKL

puisse activer NCC dans l'œuf de xénope en conditions standard alors qu'il est nécessaire d'abaisser la concentration intracellulaire de chlorure $\left(\left[\mathrm{Cl}^{-}\right]_{\mathrm{i}}\right)$ pour obtenir le même effet avec WNK4 suggère que cette dernière est plus sensible à la concentration de chlorure que L-WNKl. Cette hypothèse a été très récemment confirmée par une nouvelle étude. Terker et al ont en effet montré que l'activité kinase de WNK4 diminue progressivement lorsque la concentration intracel- lulaire en chlorure augmente de 10 à $40 \mathrm{mM}$, alors que celle de L-WNKl est stable aux mêmes concentrations et ne commence à diminuer que lorsque cette concentration atteint $60 \mathrm{mM}[27]$.

\section{Sensibilité des WNK à la concentration en chlorure et régulation de la sécrétion du potassium}

L'ensemble des données récentes que nous avons présentées a permis d'établir un nouveau modèle de régulation de NCC par WNKl et WNK4. Elles réconcilient les observations in vivo et in vitro (Figure 3 ). II reste désormais à déterminer les conditions physiologiques dans lesquelles cette voie de régulation intervient. Deux études suggèrent qu'elle pourrait être mise en jeu pour adapter l'excrétion de potassium en réponse à des modifications de la quantité de cet élément dans l'alimentation.

Plusieurs études réalisées chez le rat et la souris ont ainsi montré que l'expression de NCC varie en fonction de la teneur en potassium des aliments : elle est augmentée lorsque le régime est pauvre en potassium et diminuée lorsque le régime est riche en potassium [28] (Figure 3). La diminution de l'expression de NCC dans cette dernière condition augmente la quantité de sodium délivrée au tubule connecteur (CNT) et au canal collecteur cortical (CCD), situés en aval du DCT. Ceci stimule l'activité du canal épithélial sodique $(\varepsilon \mathrm{NaC})$, exprimé dans ces segments du néphron (Figure 1). La réabsorption de sodium par $\varepsilon \mathrm{NaC}$ est électrogénique. Elle est accompagnée d'une sécrétion de potassium par le canal potassique ROMK (renal outer medullary potassium channel), ce qui permet l'excrétion du potassium en excès (Figure I). À l'inverse, lors d'une restriction en potassium, la majo- 


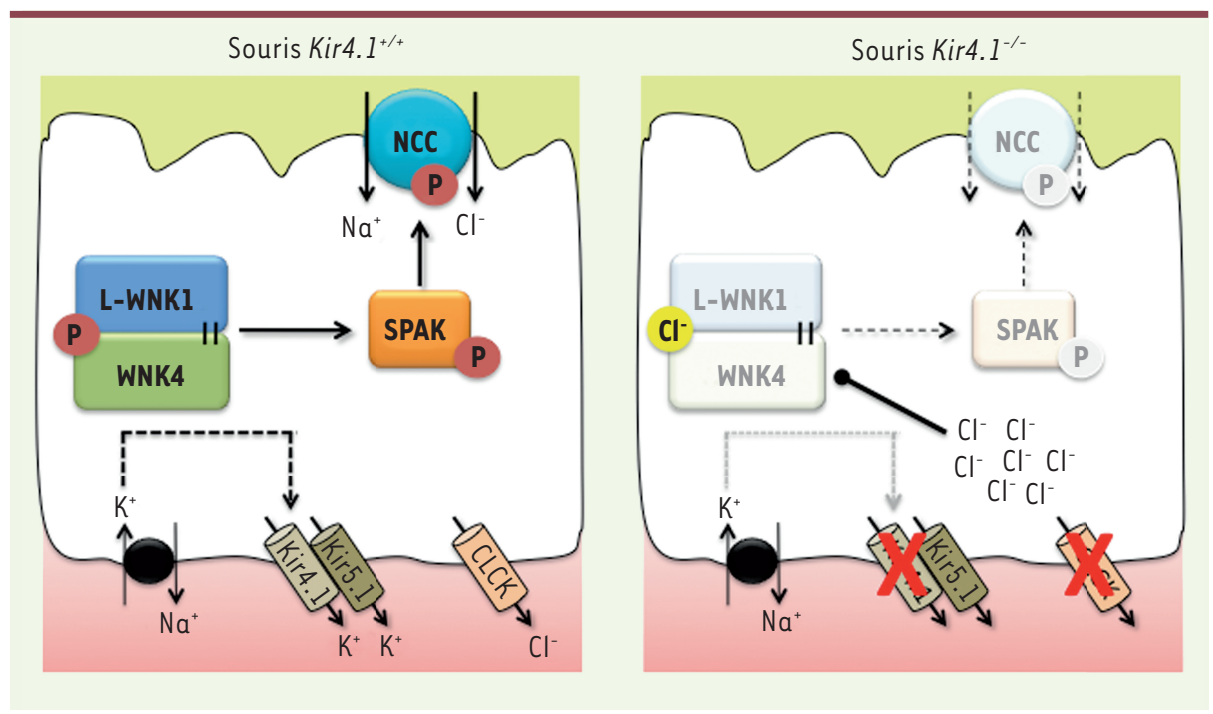

Figure 4. Modulation de la concentration intracellulaire de chlorure $\left(\left[\mathrm{Cl}^{-}\right] i\right)$ dans le tube contourné distal (DCT). Chez la souris sauvage, le canal potassique Kir4.l assure le recyclage du $\mathrm{K}^{+}$pour la pompe $\mathrm{Na}^{+} / \mathrm{K}^{+}-$ ATPase et produit le gradient électrochimique nécessaire à l'extrusion du $\mathrm{Cl}^{-}$à la membrane baso-latérale. Chez les souris mutées pour Kir4.1, l'inactivation de ce canal (panneau de droite) conduit à une augmentation de la $\left[\mathrm{Cl}^{-}\right]_{i}$ et donc à une inactivation de la cascade de phosphorylation WNK1/4-SPAKNCC. Seuls les multimères L-WNK1/WNK4 sont représentés afin de simplifier le schéma. CLCK : canal chlorure; Kir5.1 : canal potassique formant un hétérodimère fonctionnel avec Kir4.1. SPAK : Ste20-related proline-alanine rich kinase; NCC : transporteur $\mathrm{Na}^{+}-\mathrm{Cl}^{-}$.

rité du sodium est réabsorbée par NCC dans le DCT, ce qui diminue la réabsorption de sodium par $\varepsilon \mathrm{NaC}$ et la sécrétion de potassium par ROMK. Ce mécanisme pourrait expliquer l'hyperkaliémie observée chez les patients $\mathrm{FHHt}$.

La question restait de comprendre comment est contrôlé le niveau d'expression de NCC dans ces deux conditions. Terker et al. ont récemment démontré que la concentration extracellulaire de potassium $\left(\left[\mathrm{K}^{+}\right]_{\mathrm{e}}\right)$ a une influence directe sur la concentration intracellulaire de chlorure $\left(\left[\mathrm{Cl}^{-}\right]_{\mathrm{i}}\right)[29]$. Ainsi, une $\left[\mathrm{K}^{+}\right]_{\mathrm{e}}$ basse provoque une diminution de la $\left[\mathrm{Cl}^{-}\right]_{i}$ et stimule l'expression de NCC via la voie de signalisation WNKI-SPAK (Figure 3). La modification de la $\left[\mathrm{Cl}^{-}\right]_{i}$ par la $\left[\mathrm{K}^{+}\right]_{\mathrm{e}}$ fait intervenir le canal potassique Kir4.l, également appelé KCNJ10 (potassium channel, inwardly rectifying subfamily J, member 10). Ce canal est exprimé au pôle basolatéral des cellules du DCT, du CNT et du CCD. En sécrétant du potassium, ce canal assure le recyclage de cet ion pour la pompe $\mathrm{Na}^{+} / \mathrm{K}^{+}$-ATPase, qui permet la sortie du sodium réabsorbé par NCC. Cette sécrétion de potassium permet également d'établir un gradient électrochimique propice qui favorise l'efflux des ions chlorure réabsorbés par NCC via le canal chlorure CLCKb (renal chloride channel b)/Barttine, en maintenant cet ion hors de l'équilibre (Figure 4). La conductance potassique dans le tube contourné distal dépendant du seul canal Kir4.1, une modulation de l'activité de ce canal est susceptible d'influencer largement l'efflux de chlorure en faisant varier la différence de potentiel membranaire. Cette relation entre activité de Kir4.1 et efflux des ions chlorure a été récemment démontrée par l'équipe de W.H. Wang, grâce à la caractérisation d'un modèle d'inactivation génique [30]. Des expériences de patch-clamp ${ }^{9}$ ont en effet montré que la conductance au chlorure baso-latérale de DCT isolés de souris Kir4. $1^{-/-}$ est inférieure à celle des DCT de souris sauvages. Cette diminution de conductance devrait conduire à une augmentation de la concentration en chlorure intracellulaire. Si cela n'a pas été démontré chez la souris, Terker et al. ont montré que c'est le cas dans des cellules HEK293 exprimant un mutant null ${ }^{10}$ du gène Kir4.1 [29]. En conséquence, l'augmentation de la $\left[\mathrm{Cl}^{-}\right]_{i}$ devrait à son tour inhiber l'activation de SPAK par les WNK et donc l'expression et la phosphorylation de NCC (Figure 4). Cette hypothèse a été vérifiée chez la souris Kir4.1 $1^{-1}$. Ces animaux présentent en effet une diminution de l'expression de SPAK et de NCC [30]. Ces résultats permettent d'expliquer comment des mutations inactivatrices du gène codant Kir4.l peuvent être à l'origine du syndrome EAST (epilepsy, ataxia, sensorineural deafness, and tubulopathy)/SeSAME (seizures, sensorineural deafness, ataxia, mental retardation, and electrolyte imbalance), un syndrome qui touche de nombreux organes et dont les manifestations rénales sont semblables à celles observées chez les patients atteints du syndrome de Gitelman causé, lui, par des mutations inactivatrices de NCC $[31,32]$.

\section{Conclusions et perspectives}

L'ensemble de ces études a donc permis de réconcilier les données in vivo et in vitro, obtenues ces dix dernières années, sur la régulation de l'activité de NCC par les WNK. Elles ont surtout permis de commencer à comprendre comment la voie WNK-SPAK-NCC intervient en conditions physiologiques, notamment lors de variations de l'apport en potassium. II reste cependant encore de nombreux points à éclaircir pour

\footnotetext{
${ }_{9}^{9}$ La technique du patch-clamp consiste à enregistrer les sauts de courant survenant lorsque, à voltage constant, l'état d'ouverture d'un canal ionique isolé se modifie.

${ }^{10}$ Une mutation qui élimine complètement la fonction d'un gène.
} 
comprendre pleinement le rôle complexe joué par ces kinases dans la régulation coordonnée de la balance du sodium et du potassium. Une question cruciale est notamment la mesure de la concentration intracellulaire de chlorure et de ses variations dans ces différentes conditions. $\diamond$

\section{SUMMARY}

New perspective on the role of WNK1 and WNK4 in the regulation of $\mathrm{NaCl}$ reabsorption and $\mathrm{K}^{+}$secretion by the distal nephron The study of Familial Hyperkalemic Hypertension (FHHt), a rare monogenic disease, allowed remarkable advances in the understanding of the mechanisms of regulation of $\mathrm{NaCl}$ reabsorption by the distal nephron. FHHt results from mutations in the genes encoding WNKl and WNK4, two serine-threonine kinases of the WNK (With No lysine [K]) family. The clinical manifestations of FHHt are due, among others, to an increased activity of the $\mathrm{Na}^{+}-\mathrm{Cl}^{-}$cotransporter NCC. Several groups therefore tried to understand how WNKI and WNK4 could regulate NCC. However, the data were often contradictory. Two of our recent studies allowed to partially explain these controversies and to propose a new model for the regulation of NCC by the WNKs. $\diamond$

\section{LIENS D'INTÉRÊT}

Les auteurs déclarent n'avoir aucun lien d'intérêt concernant les données publiées dans cet article.

\section{RÉFÉRENCES}

1. Berghoff RS, Geraci AS. The influence of sodium chloride on blood pressure. Br Med J 1929 ; 56 : 395-7.

2. Sacks FM, Svetkey LP, Vollmer WM, et al. Effects on blood pressure of reduced dietary sodium and the dietary approaches to stop hypertension (DASH) diet. DASH-sodium collaborative research group. N Engl J Med $2001 ; 344: 3-10$.

3. Guyton AC, Coleman TG, Cowley AV, Jr, et al. Arterial pressure regulation. Overriding dominance of the kidneys in long-term regulation and in hypertension. Am J Med $1972 ; 52$ : 584-94.

4. Gordon RD, Klemm, SA, Tunny TJ et al. Gordon's syndrome: a sodium-volume-dependent form of hypertension with a genetic basis. In : Brenner JHLaBM, ed., Hypertension: patholgy, diagnosis and management. New York : Raven Press, 1995 : 2111-3.

5. Wilson FH, Disse-Nicodeme $\mathrm{S}$, Choate KA, et al. Human hypertension caused by mutations in WNK kinases. Science $2001 ; 293$ : 1107-12.

6. Xu B, English JM, Wilsbacher JL, et al. WNKl, a novel mammalian serine/threonine protein kinase lacking the catalytic lysine in subdomain II. J Biol Chem $2000 ; 275: 16795-801$.

7. Bazua-Valenti S, Chavez-Canales M, Rojas-Vega L, et al. The effect of WNK4 on the $\mathrm{Na}^{+}-\mathrm{Cl}$ cotransporter is modulated by intracellular chloride. J Am Soc Nephrol 2014 ; 26 : 1781-6.

8. Chavez-Canales M, Zhang C, Soukaseum C, et al. WNK-SPAK-NCC cascade revisited: WNK1 stimulates the activity of the $\mathrm{Na}-\mathrm{Cl}$ cotransporter via SPAK, an effect antagonized by WNK4. Hypertension 2014 ; 64 : 1047-53.

9. Wilson FH, Kahle KT, Sabath $\varepsilon$, et al. Molecular pathogenesis of inherited hypertension with hyperkalemia: the $\mathrm{Na}-\mathrm{Cl}$ cotransporter is inhibited by wild-type but not mutant WNK4. Proc Natl Acad Sci USA $2003 ; 100: 680-4$.

10. Yang $\mathrm{CL}$, Angell J, Mitchell R, Ellison DH. WNK kinases regulate thiazide-sensitive $\mathrm{Na}-\mathrm{Cl}$ cotransport. J Clin Invest $2003 ; 111: 1039-45$.

11. Delaloy C, Lu J, Houot AM, et al. Multiple promoters in the WNKl gene: one controls expression of a kidney-specific kinase-defective isoform. Mol Cell Biol $2003 ; 23: 9208-21$.

12. Vidal-Petiot $\varepsilon$, $\varepsilon$ lvira-Matelot $\varepsilon$, Mutig $K$, et al. WNK1-related familial hyperkalemic hypertension results from an increased expression of L-WNKl specifically in the distal nephron. Proc Natl Acad Sci USA 2013 ; 110 : 14366-71.

13. Golbang AP, Cope G, Hamad A, et al. Regulation of the expression of the $\mathrm{Na} / \mathrm{Cl}$ cotransporter (NCCT) by WNK4 and WNK1: evidence that accelerated dynamin-dependent endocytosis is not involved. Am J Physiol Renal Physiol 2006 ; 291 : F1369-76.

14. Moriguchi T, Urushiyama S, Hisamoto N, et al. WNKl regulates phosphorylation of cation-chloridecoupled cotransporters via the STE20-related kinases, SPAK and OSR1. J Biol Chem 2005 ; 280 : 42685-93.
15. Zambrowicz BP, Abuin A, Ramirez-Solis R, et al. Wnkl kinase deficiency lowers blood pressure in mice: a gene-trap screen to identify potential targets for therapeutic intervention. Proc Natl Acad Sci USA 2003; 100 : 14109-14.

16. Vidal-Petiot $\varepsilon$, Cheval L, Faugeroux J, et al. A new methodology for quantification of alternatively spliced exons reveals a highly tissue-specific expression pattern of WNKl isoforms. PLoS One 2012; 7 : e37751.

17. Castaneda-Bueno M, Cervantes-Perez LG, Vazquez N, et al. Activation of the renal $\mathrm{Na}^{+}: \mathrm{Cl}^{-}$cotransporter by angiotensin II is a WNK4-dependent process. Proc Natl Acad Sci USA 2012 ; 109 : 7929-34.

18. Burkhard P, Stetefeld J, Strelkov SV. Coiled coils: a highly versatile protein folding motif. Trends Cell Biol $2001 ; 11: 82-8$.

19. Boyden LM, Choi M, Choate KA, et al. Mutations in kelch-like 3 and cullin 3 cause hypertension and electrolyte abnormalities. Nature $2012 ; 482$ : 98-102.

20. Louis-Dit-Picard H, Barc J, Trujillano D, et al. KLHL3 mutations cause familial hyperkalemic hypertension by impairing ion transport in the distal nephron. Nat Genet 2012 ; $44:$ 456-60, S1-3.

21. Shibata S, Zhang J, Puthumana J, et al. Kelch-like 3 and Cullin 3 regulate electrolyte homeostasis via ubiquitination and degradation of WNK4. Proc Natl Acad Sci USA 2013 ; 110 : 7838-43.

22. Wakabayashi M, Mori T, Isobe K, et al. Impaired KLHL3-mediated ubiquitination of WNK4 causes human hypertension. Cell Rep $2013 ; 3$ : 858-68.

23. Wu G, Peng JB. Disease-causing mutations in KLHL3 impair its effect on WNK4 degradation. FEBS Lett $2013 ; 587: 2099-104$.

24. Piala AT, Moon TM, Akella R, et al. Chloride sensing by WNKl involves inhibition of autophosphorylation. Sci Signal $2014 ; 7$ : ra4l.

25. Zagorska A, Pozo-Guisado E, Boudeau J, et al. Regulation of activity and localization of the WNK1 protein kinase by hyperosmotic stress. J Cell Biol $2007 ; 176: 89-100$.

26. Boettger T, Hubner CA, Maier $\mathrm{H}$, et al. Deafness and renal tubular acidosis in mice lacking the $\mathrm{K}-\mathrm{Cl}$ co-transporter Kcc4. Nature 2002 ; 416 : 874-8.

27. Terker AS, Zhang C, Erspamer KJ, et al. Unique chloride-sensing properties of WNK4 permit the distal nephron to modulate potassium homeostasis. Kidney Int 2015 ; doi : 10.1038/ki.2015.289.

28. Vallon V, Schroth J, Lang F, et al. Expression and phosphorylation of the $\mathrm{Na}^{+}-$ $\mathrm{Cl}^{-}$cotransporter NCC in vivo is regulated by dietary salt, potassium, and SGK1. Am J Physiol Renal Physiol 2009 ; 297 : F704-12.

29. Terker AS, Zhang C, McCormick JA, et al. Potassium modulates electrolyte balance and blood pressure through effects on distal cell voltage and chloride. Cell Metab $2015 ; 21: 39-50$.

30. Zhang C, Wang L, Zhang J, et al. KCNJ10 determines the expression of the apical $\mathrm{Na}-\mathrm{Cl}$ cotransporter (NCC) in the early distal convoluted tubule (DCT1). Proc Natl Acad Sci USA 2014 ; 111 : 11864-9.

31. Reichold M, Zdebik AA, Lieberer $\varepsilon$, et al. KCNJ10 gene mutations causing EAST syndrome (epilepsy, ataxia, sensorineural deafness, and tubulopathy) disrupt channel function. Proc Natl Acad Sci USA 2010 ; 107 : 14490-5.

32. Scholl UI, Choi M, Liu T, et al. Seizures, sensorineural deafness, ataxia, mental retardation, and electrolyte imbalance (SeSAME syndrome) caused by mutations in KCNJ10. Proc Natl Acad Sci USA 2009 ; 106 : 5842-7.

33. Eladari $D$, Chambrey R, Leviel F. Identification d'une nouvelle cible des diurétiques thiazidiques dans le rein. Med Sci (Paris) $2010 ; 26: 549-52$.

34. Hadchouel J, Delaloy C, Jeunemaitre X. WNKl et WNK4, nouveaux acteurs de I'homéostasie hydrosodée. Med Sci (Paris) 2005 ; 21 : 55-60.

35. Louis-Dit-Picard H, Hadchouel J, Jeunemaitre X. KLHL3 et CULLIN-3. Med Sci (Paris) $2012 ; 28: 703-6$.
TIRÉS À PART

J. Hadchouel 